\title{
Nematic phase in stripe-forming systems within the self-consistent screening approximation
}

\author{
Daniel G. Barci, ${ }^{1}$ Alejandro Mendoza-Coto, ${ }^{2}$ and Daniel A. Stariolo ${ }^{2}$ \\ ${ }^{1}$ Departamento de Física Teórica, Universidade do Estado do Rio de Janeiro, Rua São Francisco Xavier 524, \\ 20550-013 Rio de Janeiro, Brazil \\ ${ }^{2}$ Departamento de Física, Universidade Federal do Rio Grande do Sul, CP 15051, 91501-970 Porto Alegre, RS, Brazil
}

(Received 9 September 2013; published 23 December 2013)

\begin{abstract}
We show that in order to describe the isotropic-nematic transition in stripe-forming systems with isotropic competing interactions of the Brazovskii class it is necessary to consider the next to leading order in a $1 / N$ approximation for the effective Hamiltonian. This can be conveniently accomplished within the self-consistent screening approximation. We solve the relevant equations and show that the self-energy in this approximation is able to generate the essential wave vector dependence to account for the anisotropic character of a two-point correlation function characteristic of a nematic phase.
\end{abstract}

DOI: 10.1103/PhysRevE.88.062140

\section{INTRODUCTION}

There is a large number of materials which present a tendency to form charge and/or magnetic stripe patterns. These patterns can be self-organized in many different phases, such us stripes, lamellae, bubbles, and others. Inhomogeneous phases of this type have been observed in systems as diverse as strongly correlated electronic systems [1] or ferromagnetic thin films [2]. A common property of all these systems is that there is a certain degree of frustration, coming from the lattice structure or from competing interactions.

Complex phases are generally characterized by broken symmetries manifested by the long-wavelength behavior of correlations. For instance, stripe order breaks rotational as well as translational symmetry in one direction, while crystal or bubble phases break translational invariance in all directions. Moreover, it is possible to have a phase where the the translational symmetry is restored by the proliferation of topological defects, however the system will still have orientational order. The nematic or the hexatic phases are such homogeneous phases that break rotational symmetry while preserving translation invariance [3].

In the last years there has been a renewed interest in the nematic phase, due to the fact that states with this symmetry have been observed in several highly correlated electron systems [1] such as quantum Hall systems, ruthenate compounds, cuprates, and Fe-based high temperature superconductors. Whether this phase is relevant to describe the interesting and exotic transport properties of such materials is still an open question. However, there is a growing amount of data suggesting that the physics of the nematic phase could be intimately related with the non-Fermi liquid behavior of anisotropic metal states.

Clear evidences of an electronic nematic phase appear in ultrahigh mobility two-dimensional electron systems (2DES) in $\mathrm{GaAs} / \mathrm{GaAlAs}$ heterostructures [4,5] at extremely low temperatures and moderate magnetic fields. While for huge magnetic fields the fractional quantum Hall $(\mathrm{QH})$ phase dominates the physics of the first Landau level, the nematic phase appears when the Fermi level lies near the middle of the third and higher Landau levels (smaller magnetic fields). The most evident signature of the $\mathrm{QH}$ nematic is the strong temperature dependence of anisotropic transport properties [6].
PACS number(s): 64.60.A-, 68.35.Rh, 75.70.Kw

The electronic nematic phase has also been observed in the bilayer ruthenate compound $\mathrm{Sr}_{3} \mathrm{Ru}_{2} \mathrm{O}_{7}$ at finite magnetic field $[7,8]$. While the data suggest that a metamagnetic quantum critical point can be reached by changing the direction of the applied magnetic field [9,10], transport properties of this system are strongly anisotropic and its temperature dependence is very similar to the 2DES described before.

Other examples of stripe-forming materials are the cuprates high $T_{c}$ superconductors [11]. In addition to superconductivity, typical ingredients found in systems with competing interactions, such as inhomogeneity, anisotropy, disorder, and glassiness, coexist. The intermediate state between the Mott insulator and the superconducting phase is usually understood as a spin glass with local stripe order, called "cluster glass." Although the electronic cluster glass state exhibits no known long-range order, some electronic order is always detected by local probes [12-16]. Also, fluctuating stripes have been measured [17] at the onset of the pseudogap state of $\mathrm{Bi}_{2} \mathrm{Sr}_{2} \mathrm{CaCu}_{2} \mathrm{O}_{8+x}$ using spectroscopic mapping with a scanning tunneling microscope. In the same direction, recent measurements [18] of the Nernst effect in $\mathrm{YBa}_{2} \mathrm{Cu}_{3} \mathrm{O}_{y}$ showed that the pseudogap temperature coincides with the appearance of a strong in-plane anisotropy of electronic origin, compatible with the electronic nematic phase [19].

In a completely different context, stripe domains with ferromagnetic order are observed in ultrathin magnetic films with perpendicular anisotropy [2,20-23]. For instance, in monolayers of $\mathrm{Fe}$ grown on $\mathrm{Cu}$ substrates, the local magnetization shows very complex temperature dependent striped patterns with a bunch of topological defects. A nematic order in these systems has been proposed by analyzing frustrated ferromagnetic models analytically $[24,25]$ as well as with Monte Carlo simulations [26,27].

From a theoretical point of view, the stripe phase was extensively studied in several systems and its origin is well understood. Different mean-field approaches correctly capture the physics of the stripe phase. For instance, QH stripes are correctly described by a Hartree-Fock approximation of a 2DES in a magnetic field with Coulomb interactions [28-30]. Hartree-Fock solutions of the Hubbard model [31-33], slaveboson mean-field theories of the $t-J$ model [34-36], and even studies of Coulomb frustrated phase separation [37] provide a reasonable description of the stripe phase of high $T_{c}$ 
superconductors. In ferromagnetic thin films, the stripe phase has been analyzed by means of a mean-field treatment of the frustrated Ising-dipolar model [38], by elasticity of domain walls analysis of an Heisenberg Hamiltonian [23], and through Monte Carlo simulations [26,39,40]. The hidden reason of why mean-field treatments work pretty well for the stripe phase resides in the fact that the Hamiltonian can be naturally written in terms of the stripe order parameter (i.e., local charged density or local magnetization, depending on the case).

All these systems, despite the different microscopic origin, have a natural scale that dominates the stripe modulation, originated from competing interactions. Therefore, in the spirit of the Landau theory of phase transition, it is possible to describe the stripe phase by means of a coarse-grained Hamiltonian describing a system constrained to a thin shell in momentum space around a characteristic wave vector of modulus $k_{0} \cdot{ }^{1}$ Examples of scalar and vector order parameters behaving this way were studied in a seminal work by Brazovskii [41]. He showed that in systems with a spectrum of fluctuations dominated by a shell of nonzero wave vector, there is a first-order phase transition at a finite temperature from an isotropic to a stripe phase, induced by field fluctuations. The main fluctuations were taken into account by implementing a self-consistent Hartree approximation, which is known to be equivalent to the leading order term in a $1 / N$ expansion of a system with $O(N)$ symmetry.

On the other hand, the nematic phase is more elusive. The nematic order parameter (as will be described in the following sections) is quadratic in the original degrees of freedom, therefore, it is essentially governed by the physics of fluctuations and cannot be captured by naive mean-field theory. Theoretical predictions of the nematic phase are based on the study of specific interactions written in terms of the nematic order parameter. For instance, in Fermi liquids, the isotropicnematic phase transition was studied using different techniques such as RPA [42], multidimensional bosonization [43,44], and Landau Fermi liquid theory $[45,46]$, on specific models with explicit attractive quadrupole-quadrupole interaction. Moreover, in Ref. [47] we have shown that in coarse-grained models of the Brazovskii class, the quadrupolar interaction is naturally generated, since it is relevant in the renormalization group sense. In particular, we have shown that in isotropic 2D models, although the stripe phase cannot exist since long wavelength fluctuations diverge linearly, the nematic phase can indeed exist and it is in the Kosterlitz-Thouless universality class [48].

One way of describing the isotropic-nematic phase transition is to observe spontaneous Fermi surface deformations in the case of Fermi liquids, or deformations of the high temperature form factor in the case of classical systems. Provided we begin with a Hamiltonian with local polynomial interactions, the Hartree (or Hartree-Fock for fermions)

\footnotetext{
${ }^{1}$ Of course this simple assumption is unable to capture the whole richness of the phase diagram of systems like the dipolar frustrated Ising model in a square lattice, as observed, e.g., through Monte Carlo simulations $[26,38,40]$. In this case, the tetragonal symmetry induced by the lattice should be explicitly incorporated into the Landau expansion.
}

approximation provides a constant (momentum independent) renormalization to the effective Hamiltonian and then it cannot capture any deformation in momentum space. In order to have a chance to observe relevant fluctuations associated with the isotropic-nematic phase transition it is necessary to compute corrections producing a momentum dependent self-energy. In this paper we show that the minimal approach to describe the isotropic-nematic transition in systems with isotropic competing interactions is the self-consistent screening approximation (SCSA) $[49,50]$, which is equivalent to introducing the next to leading order term in a $1 / N$ expansion of the effective Hamiltonian in a self-consistent way.

In this work we consider the simplest Brazovskii model in two dimensions and compute the correlation function in the SCSA. We show that there is a critical temperature at which the system spontaneously breaks rotational symmetry, signaling the presence of an isotropic-nematic phase transition. This definitively confirms our previous claims [47,48] based on symmetry and RG arguments within the context of a completely controlled calculation.

This paper is organized as follows: In Sec. II we introduce the model and the essential background on the nematic order parameter. In Sec. III we introduce the SCSA and compute, both numerically and analytically, the two-point correlation function leading to the isotropic-nematic transition. Section IV is devoted to the conclusions and a short discussion of our results.

\section{MODEL HAMILTONIAN, STRIPE FORMATION, AND THE NEMATIC PHASE}

We are interested in the low temperature physics of $d=2$ models with isotropic competing interactions and Ising symmetry. Universal characteristics can be well described by a coarse-grained effective Hamiltonian written in terms of a real scalar field $\phi(\vec{x})$, of which the quadratic part in reciprocal space reads

$$
H_{0}=\int_{\Lambda} \frac{d^{2} k}{(2 \pi)^{2}} \phi(\vec{k})\left[r_{0}+A\left(k-k_{0}\right)^{2}+\cdots\right] \phi(-\vec{k}),
$$

where $r_{0}(T) \sim\left(T-T^{*}\right), k=|\vec{k}|$, and $k_{0}=\left|\vec{k}_{0}\right|$ is a characteristic scale given by the competing nature of the microscopic interactions [51]. $\int_{\Lambda} d^{2} k \equiv \int_{0}^{2 \pi} d \theta \int_{k_{0}-\Lambda}^{k_{0}+\Lambda} d k k$ and $\Lambda \sim \sqrt{r_{0} / A}$ is a cut off where the expansion of the free energy up to quadratic order in the wave vector makes sense [41]. The "mass" $1 / A$ measures the curvature of the dispersion relation around the minimum $k_{0}$ and the ellipses in Eq. (1) mean higher order terms in $\left(k-k_{0}\right)$.

The structure factor or, equivalently, the two-point correlation function:

$$
G_{0}(k)=\frac{1}{r_{0}+A\left(k-k_{0}\right)^{2}}
$$

has a maximum at $k=k_{0}$ with a correlation length $\xi \sim$ $\sqrt{A / r_{0}}$. Therefore, near criticality $\left(r_{0} \rightarrow 0\right)$ the physics is dominated by an annulus in reciprocal space with wave vector $k \sim k_{0}$ and width $2 \Lambda$. This situation is quite similar to fermionic systems at low temperature, where $k_{0}$ plays the role of the Fermi momentum, and the reduction of phase space to a spherical shell (in $d=3$ ) centered at the Fermi momentum 
is ruled by the Pauli exclusion principle. The question is how interactions modify this picture. The simplest interaction term is given by a local quartic term of the form

$\mathcal{H}_{i}=v \int\left(\prod_{i=1}^{4} \frac{d^{2} k_{i}}{(2 \pi)^{2}}\right) \phi\left(\vec{k}_{1}\right) \cdots \phi\left(\vec{k}_{4}\right) \delta^{2}\left(\vec{k}_{1}+\vec{k}_{2}+\vec{k}_{3}+\vec{k}_{4}\right)$

where $v$ measures the interaction intensity. The free correlation is renormalized by the interaction term Eq. (3). The simplest correction is given by the self-consistent field approximation in which the quartic term is approximated in the form $\phi^{4}(\vec{x}) \simeq$ $\left\langle\phi^{2}(\vec{x})\right\rangle \phi^{2}(\vec{x})$. In this way the original theory is approximated by an effective one which is quadratic in the field $\phi$ and thus can be solved exactly. In this approximation, the correlation function $G(\vec{k})$ has the same free structure of Eq. (2) with a renormalized $r_{0} \rightarrow r$, given by the self-consistent equation [3]

$$
r(T)=r_{0}(T)+v T \int \frac{d^{2} k}{(2 \pi)^{2}} G(\vec{k}) .
$$

Brazovskii showed [41] that the solution of this equation drives the mean-field critical point to a fluctuation induced first-order phase transition between an isotropic and a stripe phase characterized by a modulated order parameter $\langle\phi(\vec{x})\rangle \sim \cos \left(k_{0} x\right)$. However, long wavelength fluctuations of this modulated pattern may diverge, depending on dimensionality. In $d=3$ the divergence is logarithmic in the linear size of the system leading to quasi-long-ranged stripe order, while in $d=2$ the divergence is linear implying that the system cannot order at any finite temperature.

Then, a relevant question is about the possible existence of a homogeneous phase at intermediate and low temperatures, like a nematic phase, which restores translation invariance but breaks rotational symmetry. Orientational order of this kind can be quantified by a nematic tensor order parameter given in terms of the density gradients [52]:

$$
Q_{i j} \equiv \int d^{2} x \phi(\vec{x})\left(\partial_{i} \partial_{j}-\frac{1}{2} \partial^{2} \delta_{i j}\right) \phi(\vec{x}),
$$

where $i, j=x, y$ and $\partial^{2}=\partial_{x x}+\partial_{y y}$ is the Laplacian in two dimensions. This tensor is symmetric and traceless, and in two dimensions it has only two independent elements which essentially represent the mean orientation of domain walls and the strength of the orientational order. In order to get some feeling of the physical content of this order parameter it is useful to write it in reciprocal space. After Fourier transformation and choosing the $x$ axis as the principal one, the only relevant element of the tensor is given by [52]

$$
\left\langle Q_{x x}\right\rangle=\int d^{2} k k^{2} \cos (2 \theta) G(\vec{k}),
$$

where $k_{x}=k \cos \theta, k_{y}=k \sin \theta$, and $G(\vec{k})$ is the structure factor of the system. Written in this way the orientational order parameter quantifies the degree of anisotropy of the domain pattern. In a completely isotropic phase, e.g., a liquid phase or a mosaic of domains with no preferential direction, the corresponding isotropy in the structure factor will be reflected in a zero value of the orientational order parameter. Therefore, any approximation such as the self-consistent Hartree approximation described above, leading to a constant renormalization of the correlation function, is not able to capture the physics of the nematic phase. Note that Eq. (6) acts as a filter that selects the $\cos (2 \theta)$ component of the correlation function. Then, in order to have $Q_{x x} \neq 0$, the correlation function should be anisotropic. An anisotropic form leading to a nematic phase was found in Refs. $[47,48]$, which has the form

$$
G(\vec{k})=\frac{1}{r+A\left(k-k_{0}\right)^{2}+\alpha k^{2} \cos (2 \theta)} .
$$

Here $\alpha$ is a constant which plays the role of a scalar nematic order parameter $[47,48]$. If $\alpha=0$, the correlation function is isotropic and $Q_{x x}=0$. On the other hand, for $\alpha \neq 0$, the anisotropy of the correlation function gives a finite contribution to $Q_{x x}$. Then, the nematic phase should be described by an effective quadratic Hamiltonian of the form

$$
H_{N}=\int_{\Lambda} \frac{d^{2} k}{(2 \pi)^{2}} \phi(\vec{k}) G(\vec{k})^{-1} \phi(-\vec{k}),
$$

with $G(\vec{k})$ given by Eq. (7). In other words, interactions should renormalize the Hamiltonian in a momentum-dependent way. This is only possible by at least at a two-loop approximation in a perturbative expansion. We will show in the next section that the SCSA provides the appropriate temperature dependent renormalization parameter $\alpha(T)$, being able to capture the isotropic-nematic phase transition.

\section{THE NEMATIC SOLUTION IN THE SCSA}

The set of self-consistent equations for the two-point correlation function in the SCSA are (see Supplemental Material [53])

$$
\begin{gathered}
G(\vec{k})=\frac{1}{G_{0}^{-1}(\vec{k})+\Sigma(\vec{k})}, \\
\Sigma(\vec{k})=\int \frac{d^{2} q}{(2 \pi)^{2}} D(\vec{k}-\vec{q}) G(\vec{q}), \\
D(\vec{k})=\frac{v}{1+v \Pi(\vec{k})}, \\
\Pi(\vec{k})=\int \frac{d^{2} q}{(2 \pi)^{2}} G(\vec{k}-\vec{q}) G(\vec{q}) .
\end{gathered}
$$

A general solution of this set of equations for a particular system is a formidable task. As discussed in Sec. II, we expect a solution of the form (7). Thus, our aim is to show that this set of equations admits a solution of that form, leading to an isotropic-nematic phase transition, and that the solution is stable below some critical temperature.

We work perturbatively at order $v^{2}$ in (11), such that $D(\vec{k})=$ $v-v^{2} \Pi(\vec{k})$. Then, Eqs. (10), (11), and (12) can be written in real space in the simple form

$$
\begin{gathered}
\Sigma(\vec{x})=D(\vec{x}) G(\vec{x}), \\
D(\vec{x})=v \delta(\vec{x})-v^{2} \Pi(\vec{x}), \\
\Pi(\vec{x})=G(\vec{x})^{2} .
\end{gathered}
$$


In this way the self-energy $\Sigma(\vec{x})$ is given directly as a function of $G(\vec{x})$ :

$$
\Sigma(\vec{x})=v G(0) \delta(\vec{x})-v^{2} G(\vec{x})^{3} .
$$

Then, the system of equations that actually have to be solved is reduced to

$$
\begin{aligned}
G(\vec{k}) & =\frac{1}{r_{0}+A\left(k-k_{0}\right)^{2}+\Sigma(\vec{k})}, \\
\Sigma(\vec{x}) & =v G(0) \delta(\vec{x})-v^{2} G(\vec{x})^{3},
\end{aligned}
$$

in which the first equation is written in reciprocal space and the second in real space because it is helpful in the numerical analysis.

As discussed in Sec. II, the nematic solution should be characterized by broken orientational symmetry, which in the present context means that the correlation function $G(\vec{k})$ will depend not only on the modulus of the wave vector $k$, but also on its orientation. Then, in an expansion of the self-energy around the circle of radius $k_{0}$, the lowest order form which is necessary to reveal an orientational symmetry breaking has the form $c_{1}+c_{2} \cos (2 \theta)$, where $c_{1}$ and $c_{2}$ are $k$-independent coefficients and $\theta$ is the angle relative to the direction along which the rotational symmetry is broken. This implies that only the "mass" term in the correlation function will be renormalized with a $\theta$ dependent function. Higher order terms in the self-energy will be responsible for corrections in the values of $A$ and $k_{0}$ but do not change significantly the physics near the nematic transition.

From the previous arguments we propose the following ansatz for the renormalized correlation function:

$$
G(\vec{k})=\frac{1}{r+A\left(k-k_{0}\right)^{2}+\alpha \cos (2 \theta)},
$$

where $\vec{k}=(k \cos \theta, k \sin \theta)$ and the (approximately constant) factor $k^{2} \sim k_{0}^{2}$ appearing in Eq. (7) was absorbed in $\alpha$. Note that the angular dependence of $G(\vec{k})$ with $\theta$ implies that when the symmetry is broken $(\alpha \neq 0)$ two isolated maxima appear over the $k_{y}$ axis at $k_{y}=k_{0}$ and $k_{y}=-k_{0}$, i.e., in this state the director vector of the nematic phase is along the $y$ direction in real space.

By rescaling the parameters in the form $A \rightarrow 1, k_{0} \rightarrow 1$, $\frac{r}{A k_{0}^{2}} \rightarrow r \frac{\alpha}{A k_{0}^{2}} \rightarrow \alpha, \frac{v}{A^{2} k_{0}^{2}} \rightarrow v$, the problem is expressed in a dimensionless form.

To proceed, it is useful to make a Fourier expansion of $G(k, \theta)$ given by Eq. (18). Up to second order in $\theta$ this yields

$$
\begin{aligned}
G(\vec{k})= & \frac{1}{\sqrt{\left[r+(k-1)^{2}\right]^{2}-\alpha^{2}}} \\
& \times\left\{1-\frac{2 \alpha \cos (2 \theta)}{r+(k-1)^{2}+\sqrt{\left[r+(k-1)^{2}\right]^{2}-\alpha^{2}}}\right\},
\end{aligned}
$$

where the $k$-dependent Fourier coefficients are exact. Then, the correlation function in the real space can be written as

$$
G(\vec{x})=H_{1}(x)-\alpha H_{2}(x) \cos (2 \phi),
$$

where the angle $\phi$ is such that $\phi=\pi / 2$ is along the director vector of the nematic phase and

$$
\begin{gathered}
H_{1}(x)=\int \frac{d^{2} k}{(2 \pi)^{2}} \frac{e^{i \vec{k} \cdot \vec{x}}}{\sqrt{\left[r+(k-1)^{2}\right]^{2}-\alpha^{2}}}, \\
H_{2}(x)=\int \frac{d^{2} k}{(2 \pi)^{2}} \frac{\cos (2 \theta) e^{i \vec{k} \cdot \vec{x}}}{\sqrt{\left[r+(k-1)^{2}\right]^{2}-\alpha^{2}}} \\
\times \frac{2}{r+(k-1)^{2}+\sqrt{\left[r+(k-1)^{2}\right]^{2}-\alpha^{2}}},
\end{gathered}
$$

where $\vec{k} \cdot \vec{x}=k x \cos \theta$. Then,

$$
\begin{aligned}
G(\vec{x})^{3}= & {\left[H_{1}^{3}(x)+\frac{3}{2} \alpha^{2} H_{1}(x) H_{2}^{2}(x)\right] } \\
& -\left[3 \alpha H_{1}^{2}(x) H_{2}(x)+\frac{3}{4} \alpha^{3} H_{2}^{3}(x)\right] \cos (2 \phi) .
\end{aligned}
$$

Now we are able to compute $\Sigma(\vec{k})$ by taking the inverse Fourier transform in Eq. (17). As already mentioned, we are only interested in $\Sigma\left(\vec{k}_{0}\right)$ which renormalizes the mass as a function of the angle $\theta$. Thus, we fixed $\vec{k}=\vec{k}_{0}$ and obtained $\Sigma(\vec{u})$, with $\vec{u}=\vec{k}_{0} / k_{0}$ in the rescaled variables.

The resulting renormalization equations are

$$
\begin{aligned}
r= & r_{0}+v H_{1}(0) \\
- & v^{2} \int d^{2} x\left[H_{1}^{3}(x)+\frac{3}{2} \alpha^{2} H_{1}(x) H_{2}^{2}(x)\right] e^{-i x \cos \phi}, \\
\alpha= & 3 v^{2} \int d^{2} x\left[\alpha H_{1}^{2}(x) H_{2}(x)+\frac{1}{4} \alpha^{3} H_{2}^{3}(x)\right] \\
& \times \cos (2 \phi) e^{-i x \cos \phi} .
\end{aligned}
$$

From this set of equations we can see that the solution with $\alpha=0$ is always a solution. A nonzero solution can be searched from the last equation which, after factoring out the $\alpha=0$ solution, may be written in the form

$$
\alpha=\sqrt{\frac{1-3 v^{2} \int d^{2} x H_{1}^{2}(x) H_{2}(x) \cos (2 \phi) e^{-i x \cos \phi}}{\frac{3}{4} v^{2} \int d^{2} x H_{2}^{3}(x) \cos (2 \phi) e^{-i x \cos \phi}}} .
$$

It is worth noting that this equation is not yet the solution of $\alpha$ since the functions $H_{1}(x)$ and $H_{2}(x)$ depend on $\alpha$ too through Eqs. (21) and (22), respectively. Nevertheless, this form is appropriate to proceed numerically.

\section{A. Numerical results}

In order to solve numerically Eqs. (24) and (26) it is convenient to divide the computation into two steps: First we solve the equation for $\alpha$ for a generic set of values of $r$. In this way we build the function $\alpha(r)$ which is shown in Fig. 1 for $v=0.1$. This function is independent of all parameters in the system except for $v$.

Then we proceed with the computation of $r$ varying $r_{0}$ using Eq. (24). We have to consider both cases $\alpha \neq 0$ and $\alpha=0$. In the case of $\alpha \neq 0$ we use the previously calculated function 


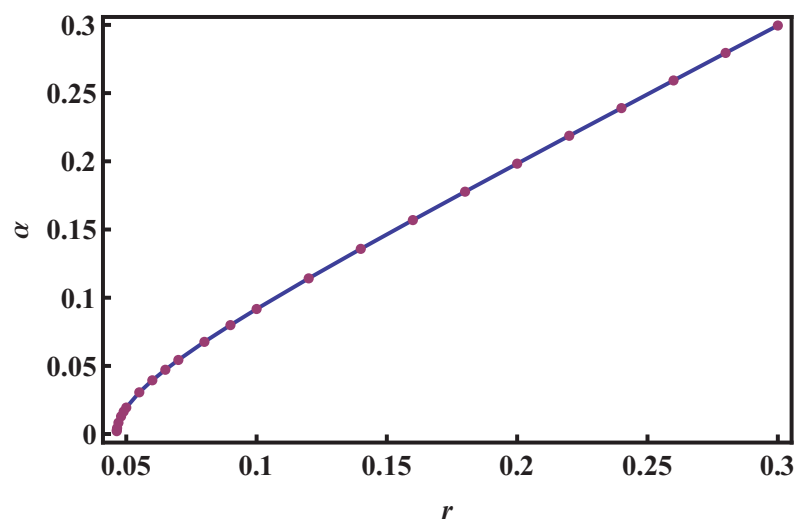

FIG. 1. (Color online) Numerical solution $\alpha(r)$ for $v=0.1$.

$\alpha(r)$ to solve Eq. (24), while for $\alpha=0$ we solve the equation for $r$ directly. In Fig. 2 we show the solution of Eq. (24) in both cases for $v=0.1$. We see how at some critical value of $r_{0}=r_{0 c}$ a bifurcation takes place and the nematic solution appears.

As usual, we consider that $r_{0}$ depends on temperature like $r_{0}=1-T$, where $T$ is the dimensionless temperature. In Fig. 3 we show the nematic order parameter as a function of temperature.

\section{B. Nature of the isotropic-nematic transition in the SCSA}

We have shown that the SCSA equations for the Brazovskii model admit a nematic solution $(\alpha \neq 0)$ as well as an isotropic one $(\alpha=0)$, as seen in Fig. 2. To establish one or the other as the thermodynamic solution we have to compare their free energies. The free energy within the SCSA is given by (see Supplemental Material [53])

$$
\begin{aligned}
F(T)= & \frac{1}{2} \int \frac{d^{2} k}{(2 \pi)^{2}} \ln \left[G^{-1}(\vec{k})\right]+\frac{1}{2} \int \frac{d^{2} k}{(2 \pi)^{2}} \ln \left[D^{-1}(\vec{k})\right] \\
& -\frac{1}{2} \int \frac{d^{2} k}{(2 \pi)^{2}} \Sigma(\vec{k}) G(\vec{k}) .
\end{aligned}
$$

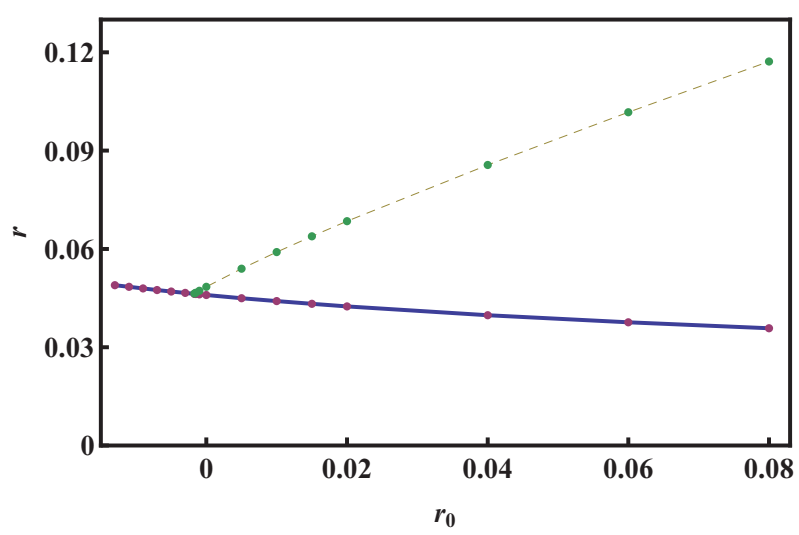

FIG. 2. (Color online) Numerical solution of $r$ as function of $r_{0}$ for $v=0.1$. The upper (dashed) line corresponds to the the solution with $\alpha \neq 0$, while the lower (full) line corresponds to the $\alpha=0$ solution.

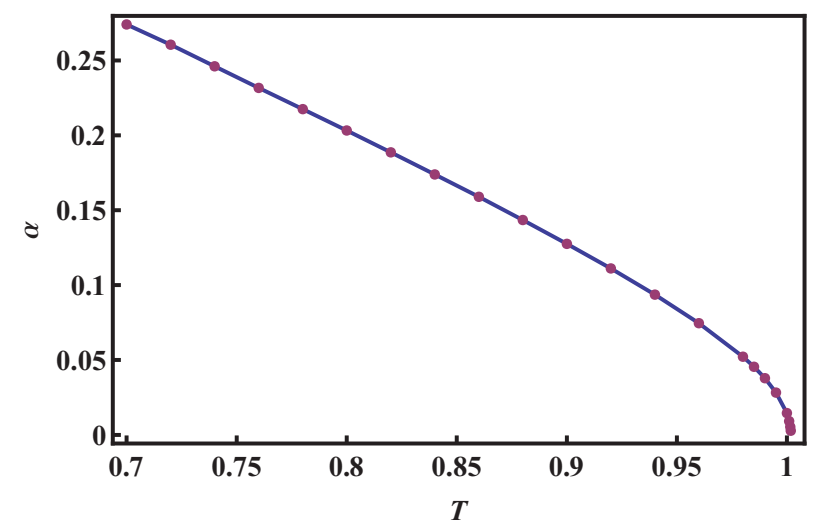

FIG. 3. (Color online) The nematic order parameter $\alpha$ as a function of the dimensionless temperature $T$ for $v=0.1$.

Expanding the logarithm in the second integral to linear order in the polarization function leads to

$$
\begin{aligned}
F(T)= & \frac{1}{2} \int \frac{d^{2} k}{(2 \pi)^{2}} \ln \left[G^{-1}(\vec{k})\right]+\frac{v}{2} \int \frac{d^{2} k}{(2 \pi)^{2}} \Pi(\vec{k}) \\
& -\frac{1}{2} \int \frac{d^{2} k}{(2 \pi)^{2}} \Sigma(\vec{k}) G(\vec{k})-\frac{1}{2} \int \frac{d^{2} k}{(2 \pi)^{2}} \ln v,
\end{aligned}
$$

where the last term in Eq. (28) is a constant. The second term of the last equation is simply given by $\int \frac{d^{2} k}{(2 \pi)^{2}} \Pi(\vec{k})=G^{2}(0)$. The term including the self-energy can be calculated considering that the function $G(\vec{k})$ is peaked at $k_{0}$. Then it is enough to consider $\Sigma(\vec{k})$ as varying only over the circle of radius $k_{0}$. The above considerations lead to a difference between the free energy of the isotropic and the nematic solutions given by

$$
\begin{aligned}
\Delta F= & F(\alpha=0)-F(\alpha \neq 0) \\
= & \frac{1}{2} \int \frac{d^{2} k}{(2 \pi)^{2}} \ln \left[\frac{G_{\alpha=0}^{-1}(\vec{k})}{G_{\alpha \neq 0}^{-1}(\vec{k})}\right]+\frac{v}{2}\left[G_{\alpha=0}^{2}(0)-G_{\alpha \neq 0}^{2}(0)\right] \\
& -\frac{1}{2} \int \frac{d^{2} k}{(2 \pi)^{2}}\left[\Sigma_{\alpha=0}(\vec{u}) G_{\alpha=0}(\vec{k})-\Sigma_{\alpha \neq 0}(\vec{u}) G_{\alpha \neq 0}(\vec{k})\right],
\end{aligned}
$$

where $\vec{u}=\vec{k} / k$.

All magnitudes involved in $\Delta F$ have already been found and then a numeric evaluation is straightforward. The results are shown in Fig. 4 for $v=0.1$. We see that for $T<T_{c}$, corresponding to $r_{0}=r_{0 c}$, the difference in the free energy $\Delta F>0$, meaning that the nematic solution presents smaller free energy than the isotropic one. We have also confirmed this result by performing an analytical expansion of the free energy near the critical point. This fact establishes the existence of a continuous isotropic-nematic transition in the context of the SCSA.

\section{Critical behavior}

To analyze the behavior near the critical point in more detail it is convenient to include the parameters $\alpha$ and $r$ in the notations of the functions $H_{1}(x) \equiv H_{1}(x, r, \alpha)$ and $H_{2}(x) \equiv$ $H_{2}(x, r, \alpha)$. Equation (26) for $\alpha=0$ provides a condition to determine the critical value of $r=r_{c}$, and Eq. (24) allows us 


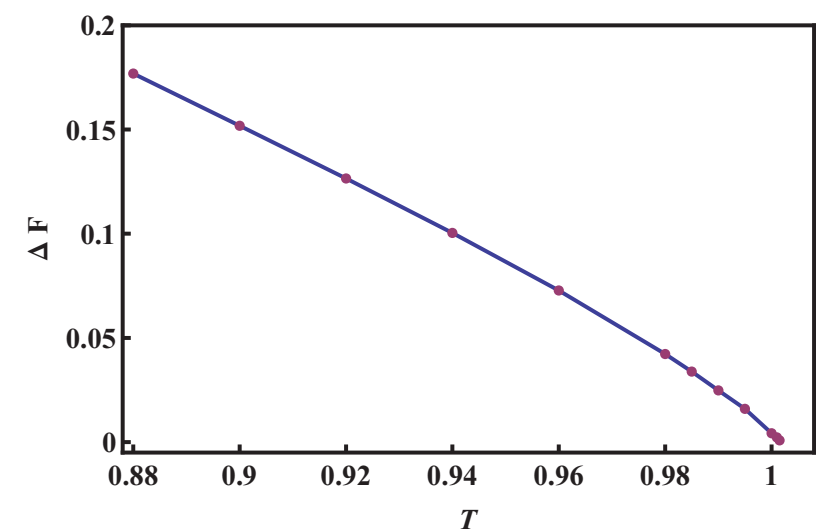

FIG. 4. (Color online) Free energy difference between the isotropic and the nematic solutions as function of dimensionless temperature for $v=0.1$.

to obtain the corresponding critical $r_{0}=r_{0 c}$ :

$$
3 v^{2} \int d^{2} x H_{1}^{2}\left(x, r_{c}, 0\right) H_{2}\left(x, r_{c}, 0\right) \cos (2 \phi) e^{-i x \cos \phi}=1
$$

and

$r_{0 c}=r_{c}-v H_{1}\left(0, r_{c}, 0\right)+v^{2} \int d^{2} x H_{1}^{3}\left(x, r_{c}, 0\right) e^{-i x \cos \phi}$.

Expanding $H_{1}$ and $H_{2}$ to leading order in $r$ and $\alpha$ around the critical point results in

$$
\begin{aligned}
& H_{1}(x, r, \alpha)=H_{1}\left(x, r_{c}, 0\right)-I_{11}(x) \Delta r+I_{12}(x) \alpha^{2}, \\
& H_{2}(x, r, \alpha)=H_{2}\left(x, r_{c}, 0\right)-I_{21}(x) \Delta r+I_{22}(x) \alpha^{2},
\end{aligned}
$$

where $\Delta r=\left(r-r_{c}\right)$ and

$$
\begin{aligned}
& I_{11}(x)=\int \frac{d^{2} k}{(2 \pi)^{2}} \frac{e^{i \vec{k} \cdot \vec{x}}}{\left[r_{c}+(k-1)^{2}\right]^{2}}, \\
& I_{12}(x)=\frac{1}{2} \int \frac{d^{2} k}{(2 \pi)^{2}} \frac{e^{i \vec{k} \cdot \vec{x}}}{\left[r_{c}+(k-1)^{2}\right]^{3}}, \\
& I_{21}(x)=2 \int \frac{d^{2} k}{(2 \pi)^{2}} \frac{e^{i \vec{k} \cdot \vec{x}} \cos (2 \theta)}{\left[r_{c}+(k-1)^{2}\right]^{3}}, \\
& I_{22}(x)=\frac{3}{4} \int \frac{d^{2} k}{(2 \pi)^{2}} \frac{e^{i \vec{k} \cdot \vec{x}} \cos (2 \theta)}{\left[r_{c}+(k-1)^{2}\right]^{4}} .
\end{aligned}
$$

Using these expansions in Eqs. (24) and (26) for $r$ and $\alpha$ we arrive at $\alpha=\sqrt{K \Delta r}$ with $\Delta r=m\left(r_{0}-r_{0 c}\right)$, where

$$
\begin{aligned}
K= & \frac{\int d^{2} x e^{-i x \cos \phi} \cos (2 \phi)\left[2 H_{1}\left(x, r_{c}, 0\right) H_{2}\left(x, r_{c}, 0\right) I_{11}(x)+H_{1}\left(x, r_{c}, 0\right)^{2} I_{21}(x)\right]}{\int d^{2} x e^{-i x \cos \phi} \cos (2 \theta)\left[\frac{1}{4} H_{2}\left(x, r_{c}, 0\right)^{3}+2 H_{1}\left(x, r_{c}, 0\right) H_{2}\left(x, r_{c}, 0\right) I_{12}(x)+H_{1}\left(x, r_{c}, 0\right)^{2} I_{22}(x)\right]}, \\
m^{-1}= & 1+v I_{11}(0)-v K I_{12}(0) \\
& -3 v^{2} \int d^{2} x e^{-i x \cos \phi}\left[H_{1}\left(x, r_{c}, 0\right)^{2} I_{11}(x)+K H_{1}\left(x, r_{c}, 0\right)^{2} I_{12}(x)+\frac{K}{2} H_{1}\left(x, r_{c}, 0\right) H_{2}\left(x, r_{c}, 0\right)^{2}\right] .
\end{aligned}
$$

$K$ and $m$ are positive numbers leading to the numerical solutions shown in Figs. 1 and 2. Both figures show that $\alpha(r)$ and $r\left(r_{0}\right)$ are increasing functions of $r$ and $r_{0}$, respectively. A direct conclusion of this calculation is that $\alpha=\sqrt{m K\left(T_{c}-T\right)}$ near the critical temperature, as is observed in Fig. 3. Then, as already found from the analysis of the free energy, the SCSA predicts a continuous second-order isotropic-nematic transition in agreement with our previous results in Refs. [47,48]. In Ref. [48] we showed that considering angular fluctuations of the nematic order parameter drives this transition to be of KT type.

\section{CONCLUSIONS}

The main result of this work has been to prove the existence of a isotropic-nematic continuous transition in a stripe-forming system within a two-loop expansion in the self-consistent screening approximation. In previous works the transition in the class of models studied here had been found by including explicitly an interaction term in the effective Hamiltonian, ruled by symmetry considerations on the nematic phase. In that case, an additional phenomenological interaction parameter was introduced in the spirit of a Landau expansion. Although the results were satisfactory from a physics point of view, showing the presence of a phase transition to a nematic phase with broken rotational symmetry in qualitative agreement with observations in several systems described by the effective Hamiltonian, an important question remained to be answered: Was it possible to obtain that interaction term from a more microscopic, or fundamental Hamiltonian? Here we have answered that question. The relevant interaction term can be obtained in a perturbative expansion of the high temperature effective $\phi^{4}$ Hamiltonian with the usual constant interaction parameter $v$, by going at least to a two-loop order, i.e., the renormalized interaction which is responsible for the appearance of a nematic phase is $O\left(v^{2}\right)$. It is now easy to see why this is so. The most simple meanfield approximations, or the one-loop Hartree approximation for the density correlations, are not able to account for a nematic phase. The physical reason lies in the anisotropy of correlations inherent to the nematic phase. Then, any renormalization independent of wave vector will preserve the rotation invariance of the high temperature correlations. In order that the self-energy correction have a $\vec{k}$ dependence which may lead to a broken rotational symmetry, one has to go beyond Hartree approximation.

We have found a breaking of rotational symmetry within the self-consistent screening approximation. Already at this level of perturbation of the original Hamiltonian the equations which determine the two-point correlation function are very 
difficult to solve in a closed form. Making a physically motivated assumption on the form of the simplest possible solution, we have solved the equations analytically near the transition and we have implemented an efficient way to solve them numerically away from the critical region. Our present results are in complete agreement with our previous results based on a Landau expansion. In particular, the isotropic-nematic transition is continuous with meanfield critical exponent of the nematic order parameter $\alpha \sim$ $\left(T_{c}-T\right)^{1 / 2}$. Note that, although the SCSA already accounts for fluctuation terms in the density field $\phi(\vec{x})$, the fact that the nematic order parameter is proportional to correlation functions implies that the present approach gives only a mean-field description of the nematic phase. As found by us in previous work, we expect this transition to be of the
Kosterlitz-Thouless type, i.e., a transition driven by the proliferation of topological defects, upon incorporation of relevant fluctuation terms in the mean-field-like nematic solution. Although anisotropic phases as described in this work have been reported in many experimental studies, as discussed in the Introduction, as far as we know the experimental characterization of the isotropic-nematic phase transitions is still a big challenge.

\section{ACKNOWLEDGMENTS}

The Brazilian agencies Fundação de Amparo à Pesquisa do Estado do Rio de Janeiro (FAPERJ) and Conselho Nacional de Desenvolvimento Científico e Tecnológico $(\mathrm{CNPq})$ are acknowledged for partial financial support.
[1] E. Fradkin, S. A. Kivelson, M. J. Lawler, J. P. Eisenstein, and A. P. Mackenzie, Annu. Rev. Condens. Matter Phys. 1, 153 (2010).

[2] N. Saratz, A. Lichtenberger, O. Portmann, U. Ramsperger, A. Vindigni, and D. Pescia, Phys. Rev. Lett. 104, 077203 (2010).

[3] P. M. Chaikin and T. C. Lubensky, Principles of Condensed Matter Physics (Cambridge University Press, Cambridge, UK, 1995).

[4] M. P. Lilly, K. B. Cooper, J. P. Eisenstein, L. N. Pfeiffer, and K. W. West, Phys. Rev. Lett. 82, 394 (1999).

[5] R. Du, D. Tsui, H. Stormer, L. Pfeiffer, K. Baldwin, and K. West, Solid State Commun. 109, 389 (1999).

[6] E. Fradkin, S. A. Kivelson, E. Manousakis, and K. Nho, Phys. Rev. Lett. 84, 1982 (2000).

[7] R. A. Borzi, S. A. Grigera, J. Farrell, R. S. Perry, S. J. S. Lister, S. L. Lee, D. A. Tennant, Y. Maeno, and A. P. Mackenzie, Science 315, 214 (2007).

[8] H. Doh, Y. B. Kim, and K. H. Ahn, Phys. Rev. Lett. 98, 126407 (2007).

[9] R. S. Perry, L. M. Galvin, S. A. Grigera, L. Capogna, A. J. Schofield, A. P. Mackenzie, M. Chiao, S. R. Julian, S. I. Ikeda, S. Nakatsuji, Y. Maeno, and C. Pfleiderer, Phys. Rev. Lett. 86, 2661 (2001).

[10] S. A. Grigera, R. S. Perry, A. J. Schofield, M. Chiao, S. R. Julian, G. G. Lonzarich, S. I. Ikeda, Y. Maeno, A. J. Millis, and A. P. Mackenzie, Science 294, 329 (2001).

[11] S. A. Kivelson, I. P. Bindloss, E. Fradkin, V. Oganesyan, J. M. Tranquada, A. Kapitulnik, and C. Howald, Rev. Mod. Phys. 75, 1201 (2003).

[12] J. H. Cho, F. Borsa, D. C. Johnston, and D. R. Torgeson, Phys. Rev. B 46, 3179 (1992).

[13] C. Panagopoulos, J. L. Tallon, B. D. Rainford, T. Xiang, J. R. Cooper, and C. A. Scott, Phys. Rev. B 66, 064501 (2002).

[14] H.-J. Grafe, N. J. Curro, M. Hücker, and B. Büchner, Phys. Rev. Lett. 96, 017002 (2006)

[15] Y. Kohsaka, C. Taylor, K. Fujita, A. Schmidt, C. Lupien, T. Hanaguri, M. Azuma, M. Takano, H. Eisaki, H. Takagi, S. Uchida, and J. C. Davis, Science 315, 1380 (2007).

[16] M. J. Lawler, K. Fujita, J. Lee, A. R. Schmidt, Y. Kohsaka, C. K. Kim, H. Eisaki, S. Uchida, J. C. Davis, J. P. Sethna, and E.-A. Kim, Nature (London) 466, 347 (2010).
[17] C. V. Parker, P. Aynajian, E. H. da Silva Neto, A. Pushp, S. Ono, J. Wen, Z. Xu, G. Gu, and A. Yazdani, Nature (London) 468, 677 (2010).

[18] R. Daou, J. Chang, D. LeBoeuf, O. Cyr-Choiniere, F. Laliberte, N. Doiron-Leyraud, B. J. Ramshaw, R. Liang, D. A. Bonn, W. N. Hardy, and L. Taillefer, Nature (London) 463, 519 (2010).

[19] S. A. Kivelson, E. Fradkin, and V. J. Emery, Nature (London) 393, 550 (1998).

[20] C. Won, Y. Z. Wu, J. Choi, W. Kim, A. Scholl, A. Doran, T. Owens, J. Wu, X. F. Jin, and Z. Q. Qiu, Phys. Rev. B 71, 224429 (2005).

[21] O. Portmann, A. Vaterlaus, and D. Pescia, Nature (London) 422, 701 (2003).

[22] A. Vaterlaus, C. Stamm, U. Maier, M. G. Pini, P. Politi, and D. Pescia, Phys. Rev. Lett. 84, 2247 (2000).

[23] A. Abanov, V. Kalatsky, V. L. Pokrovsky, and W. M. Saslow, Phys. Rev. B 51, 1023 (1995).

[24] D. G. Barci and D. A. Stariolo, Phys. Rev. B 84, 094439 (2011).

[25] D. G. Barci, L. Ribeiro, and D. A. Stariolo, Phys. Rev. E 87, 062119 (2013)

[26] S. A. Cannas, M. F. Michelon, D. A. Stariolo, and F. A. Tamarit, Phys. Rev. B 73, 184425 (2006).

[27] L. Nicolao and D. A. Stariolo, Phys. Rev. B 76, 054453 (2007).

[28] A. A. Koulakov, M. M. Fogler, and B. I. Shklovskii, Phys. Rev. Lett. 76, 499 (1996).

[29] M. M. Fogler, A. A. Koulakov, and B. I. Shklovskii, Phys. Rev. B 54, 1853 (1996).

[30] R. Moessner and J. T. Chalker, Phys. Rev. B 54, 5006 (1996).

[31] K. Machida, Physica C: Superconductivity 158, 192 (1989).

[32] H. J. Schulz, J. Phys. France 50, 2833 (1989).

[33] J. Zaanen and O. Gunnarsson, Phys. Rev. B 40, 7391 (1989).

[34] G. Seibold, E. Sigmund, and V. Hizhnyakov, Phys. Rev. B 57, 6937 (1998)

[35] J. Han, Q.-H. Wang, and D.-H. Lee, Int. J. Mod. Phys. B 15, 1117 (2001)

[36] J. Lorenzana and G. Seibold, Phys. Rev. Lett. 89, 136401 (2002).

[37] V. Emery and S. Kivelson, Physica C: Superconductivity 209, 597 (1993).

[38] S. A. Pighín and S. A. Cannas, Phys. Rev. B 75, 224433 (2007).

[39] S. A. Cannas, D. A. Stariolo, and F. A. Tamarit, Phys. Rev. B 69, 092409 (2004) 
[40] E. Rastelli, S. Regina, and A. Tassi, Phys. Rev. B 76, 054438 (2007).

[41] S. A. Brazovskii, Sov. Phys. JETP 41, 85 (1975).

[42] V. Oganesyan, S. A. Kivelson, and E. Fradkin, Phys. Rev. B 64, 195109 (2001).

[43] D. G. Barci and L. E. Oxman, Phys. Rev. B 67, 205108 (2003).

[44] M. J. Lawler, D. G. Barci, V. Fernández, E. Fradkin, and L. Oxman, Phys. Rev. B 73, 085101 (2006).

[45] J. Nilsson and A. H. Castro Neto, Phys. Rev. B 72, 195104 (2005).

[46] D. G. Barci and D. Reyes, Phys. Rev. B 87, 075147 (2013).
[47] D. G. Barci and D. A. Stariolo, Phys. Rev. Lett. 98, 200604 (2007).

[48] D. G. Barci and D. A. Stariolo, Phys. Rev. B 79, 075437 (2009).

[49] A. J. Bray, Phys. Rev. Lett. 32, 1413 (1974).

[50] A. Bray, J. Stat. Phys. 11, 29 (1974).

[51] M. Seul and D. Andelman, Science 267, 476 (1995).

[52] D. A. Stariolo and D. G. Barci, J. Phys.: Conf. Ser. 246, 012021 (2010).

[53] See Supplemental Material at http://link.aps.org/supplemental/ 10.1103/PhysRevE.88.062140 for steps which lead to the selfconsistent screening approximation. 